\title{
A hard and variable X-ray emission from the massive emission line star HD 157832
}

\author{
R. Lopes de Oliveira \\ Universidade de São Paulo, Instituto de Física de São Carlos, Caixa Postal 369, \\ 13560-970, São Carlos, SP, Brazil \\ and \\ C. Motch \\ Université de Strasbourg, CNRS UMR 7550, Observatoire Astronomique, 11 rue de \\ l'Université, F67000 Strasbourg, France
}

\begin{abstract}
We report the discovery of a hard-thermal $(\mathrm{T} \sim 130 \mathrm{MK})$ and variable $\mathrm{X}$-ray emission from the Be star HD 157832, a new member of the puzzling class of $\gamma$-Cas-like Be/X-ray systems. Recent optical spectroscopy reveals the presence of a large/dense circumstellar disc seen at intermediate/high inclination. With a B1.5V spectral type, HD 157832 is the coolest $\gamma$-Cas analog known. In addition, its non detection in the ROSAT all-sky survey shows that its average soft Xray luminosity varied by a factor larger than $\sim 3$ over a time interval of $14 \mathrm{yr}$. These two remarkable features, "low" effective temperature and likely high X-ray variability turn HD 157832 into a promising object for understanding the origin of the unusually high temperature X-ray emission in these systems.
\end{abstract}

Subject headings: stars: emission-line, Be — stars: individual (HD 157832) — X-rays: stars

\section{Introduction}

Massive stars emit thin thermal soft X-rays characterized by a plasma temperature of $\sim$ $6 \mathrm{MK}$ ( $\equiv k T \sim 0.5 \mathrm{keV}$ ), most likely due to the thermalization of part of the fast stellar wind in shocks (e.g., Güdel \& Nazé 2009). Hotter X-ray temperatures are observed in massive colliding wind binaries ( $23 \mathrm{MK}$; e.g., de Becker et al. 2006) and in the case of magnetically channeled wind shocks (up to $\sim 45 \mathrm{MK}$; e.g., Donati \& Landstreet 2009). However, the X-ray 
emission of the Be star $\gamma$ Cassiopeiae $(\gamma$-Cas) is at variance with that of other massive stars: it is dominated by a very hot component $(\mathrm{T} \sim 140-165 \mathrm{MK})$, with minor contributions of 2 or 3 colder plasmas having temperatures in the range from 1 MK to 35 MK (Smith et al. 2004; Lopes de Oliveira et al. 2010). Moreover, $\gamma$-Cas displays a moderately high X-ray luminosity $\left(10^{32-33} \mathrm{erg} \mathrm{s}^{-1} ; 0.2-12 \mathrm{keV}\right)$ variable on time scales ranging from a few seconds to several weeks, in contrast with the nearly stable flux and one order of magnitude lower luminosity usually observed from massive stars of similar spectral types.

In spite of being one of the most frequently observed stars in X-rays, the nature of the high energy emission of $\gamma$-Cas remains a puzzle. On one hand, its X-ray spectrum resembles that emitted by many cataclysmic variables, supporting the idea that accretion onto a white dwarf is responsible for the high energy emission. The thin thermal nature of the X-ray spectrum of $\gamma$-Cas is unlike the non thermal power-law energy distribution typical of all known accreting neutron stars Be/X-ray binaries and thus hints at a different kind of compact object. On the other hand, some indirect evidence of magnetic activity and simultaneous variations of the X-ray, optical, and UV emission support the idea that its X-rays are produced by magnetic reconnection at the interface between the photosphere and the inner part of the disc (Smith \& Robinson 2002).

$\gamma$-Cas remained a unique object for about 20 years. The recent discovery of at least six new $\gamma$-Cas analogs in X-ray surveys (Motch et al. 2007; Lopes de Oliveira et al. 2006, 2007; Smith \& Balona 2006; Safi-Harb et al. 2007; Rakowski et al. 2006) now allows us to find additional clues to the X-ray emission mechanism. We report here on the discovery of a new and peculiar $\gamma$-Cas analog, the Be star HD 157832.

\section{X-ray and Optical Observations}

HD 157832 was serendipitously observed by the EPIC cameras on-board the XMMNewton observatory during 21.9 ks on 2008 September 5 (ObsID 0551020101). Its X-ray counterpart is the bright source 2XMMi J172754.8-470134. All EPIC cameras were operated in the full frame mode using the medium filter which efficiently rejected optical light from the target of the observation GJ 674 and from HD 157832. The background flare-free good time interval is $17.6 \mathrm{ks}$ for the PN camera and $21.7 \mathrm{ks}$ for the MOS1 and MOS2 cameras. The data were reprocessed and reduced with Science Analysis System (SAS) software v10.

We obtained optical spectra of HD 157832 on 2010 November 9 with the Coudé spectro-

graph mounted on the Brazilian 1.6-m Perkin-Elmer telescope at the Pico dos Dias Observa- 
tory 1 Two position angles of the $600 \mathrm{l} / \mathrm{mm}$ grating allowed us to cover the blue (3960-5100 $\AA$; two exposures) and the $\mathrm{H} \alpha$ (5900-7000 $\AA$; one spectrum) regions, with a dispersion of $0.25 \AA /$ pixel. Data reduction was done with the MIDAS/ESO software following standard procedures.

\section{Results}

\subsection{Optical properties}

HD 157832 is a bright star $(\mathrm{V} \sim 6.6 \mathrm{mag})$ which has so far barely attracted attention. A rather large range of spectral types are reported in the literature: B0Vne (Buscombe \& Foster 1995), B3IVe (Jaschek \& Jaschek 1992), and B5Vnne (Thackeray et al. 1973). Our optical spectrum (see Fig. 1) shows, indeed, somewhat ambiguous signatures. The lack of Si IV $\lambda 4089$ line indicates a spectral type later than B1V, or later than B2V from the weak Si III $\lambda 4552$ line. We note, however, that the relatively low signal-to-noise of the spectrum casts some doubt on the absence or on the detection of these two lines. This is particularly true for the Si III $\lambda 4552$ which is placed between the relatively intense emission lines of Fe I $\lambda 4549$ and Fe I $\lambda 4556$. The presence of a strong $\mathrm{Mg}$ II $\lambda 4481$ line would also hint at a type later than B2V. However, the presence of C II $\lambda 4267$ indicates a B1.5V-B2V type and that of O II $\lambda 4070$ line excludes types later than B2. In addition, the intense C III $\lambda 4650$ and O II $\lambda 4640$ absorption lines argue in favor of a spectral type hotter than B1V. A luminosity class $\mathrm{V}$ is consistent with the weakness of Si IV $\lambda 4089$ line. To summarize, we constrain the spectral type to be between B0.5 and B2.5 with a most probable B1.5Ve type.

Other conspicuous features in the spectrum of HD 157832 are the intense Fe and $\mathrm{H}$ emission lines (especially $\mathrm{H} \alpha$, with an equivalent width $(\mathrm{EW}) \sim 25 \AA$ ) which reveal the high density and large extent of the circumstellar disc. The equatorial plane of the circumstellar disc, and therefore also that of the star, are likely seen at intermediate-to-high inclination, as evidenced by the double peaked profiles seen in several emission lines (e.g., Fe II $\lambda 4233$, 4584, 5018; and H $\alpha$; see Fig. 1). Using the FWHM of He I lines and the scaling relations derived by Steele et al. (1999), we obtain vsini $\sim 266 \mathrm{~km} \mathrm{~s}^{-1}$ from He I $\lambda 4471$, and vsini $~$ $217 \mathrm{~km} \mathrm{~s}^{-1}$ from He I $\lambda 4026,4143,4387$.

We built an overall spectral energy distribution using IUE LWP and SWP spectra acquired on September 121995 and Tycho, 2MASS (1.2-2.2 $\mu$ ), AKARI (9-18 $\mu$ ) and IRAS $(12-60 \mu$ ) photometry. Fitting the $2200 \AA$ bump together with the B \& V band photometry

\footnotetext{
${ }^{1}$ administered by the National Astrophysics Laboratory (LNA; Brazil)
} 
gives $\mathrm{E}(\mathrm{B}-\mathrm{V})=0.24 \pm 0.01$ (in agreement with the value of 0.25 obtained by Kozok 1985) and $T_{e f f}=25,000 \pm 1,000 \mathrm{~K}$. To that end, we used the reddening relation of Cardelli et al. (1989) and model atmospheres of Castelli \& Kurucz (2004). The range of effective temperatures points at the same B1.5Ve spectral type as derived from our optical spectrum (Bessell et al. 1998). Redwards of $1.2 \mu$, the contribution of the circumstellar disc is clearly detected as a flux excess above stellar photospheric emission.

Assuming a B1.5Ve spectral type, $M_{V}=-2.8$ mag (Humphreys \& McElrov 1984), and $\mathrm{E}(\mathrm{B}-\mathrm{V})=0.24$, we estimate a distance of 530 pc for HD 157832 .

\subsection{X-ray spectral analysis}

Spectral analysis was carried out with $X S P E C$ v12.6.0. The EPIC spectrum displays a strong FeK $\alpha$ complex in emission with an intensity consistent with that expected from a hot thin thermal emission (see Fig. 2). Rough estimates of the equivalent widths are: EW(FeK) $\sim 47 \mathrm{eV}, \mathrm{EW}(\mathrm{Fe} \mathrm{XXV}) \sim 102 \mathrm{eV}$, and $\mathrm{EW}(\mathrm{Fe} X X V I) \sim 36 \mathrm{eV}$. The intensity of the ionized Fe XXV (6.67 keV) and Fe XXVI (6.97 keV) lines and continuum, especially the hard continuum, are well described by a simple 1-T (mekal) model multiplied by a photoelectric absorption component (phabs): $k T=10.01_{-1.17}^{+1.42} \mathrm{keV}$ and $N_{H}=2.1 \pm 0.1 \times 10^{21} \mathrm{~cm}^{-2}$, with $\chi_{\nu}^{2} /$ d.o.f. $=$ 1.04/321. Abundances are consistent with solar values. However, the 1-T model underpredicts the emission observed in the $\sim 0.8-1.2 \mathrm{keV}$ range which contains several FeL-shell emission lines, and slightly over-predicts the soft $\mathrm{E}<0.7 \mathrm{keV}$ emission (see Fig. 2). The fit in both regions is clearly improved by including a second thermal component. Although the decrease of the $\chi_{\text {red }}^{2}$ from 1.04 to 0.94 is modest, it is statistically significant (F-test null hypothesis probability of $\left.3.3 \times 10^{-8}\right)$. Both plasmas have abundances consistent with solar values: there is no evidence of a sub-solar Fe abundance similar to that observed in $\gamma$-Cas (Smith et al. 2004; Lopes de Oliveira et al. 2010). A Gaussian line with $\sigma=10 \mathrm{eV}$ and centered at $6.4 \mathrm{keV}$ was added to account for the tentative Fe $\mathrm{K}$ fluorescence line. The $\mathrm{X}$-ray column density is about twice that predicted from the $\mathrm{E}(\mathrm{B}-\mathrm{V})$ excess, indicating the presence of local photoelectric absorption.

Similarly to other $\gamma$-Cas-like stars, the X-ray spectrum of HD 157832 is dominated by the hot thin thermal component which accounts for $\sim 97 \%$ of the total flux and emission measure (Table 1). The total unabsorbed flux of $3.8 \times 10^{-12} \mathrm{erg} \mathrm{cm}^{-2} \mathrm{~s}^{-1}(0.2-12 \mathrm{keV})$ corresponds to a luminosity of $\sim 1.3 \times 10^{32} \mathrm{erg} \mathrm{s}^{-1}$ at $530 \mathrm{pc}$. 


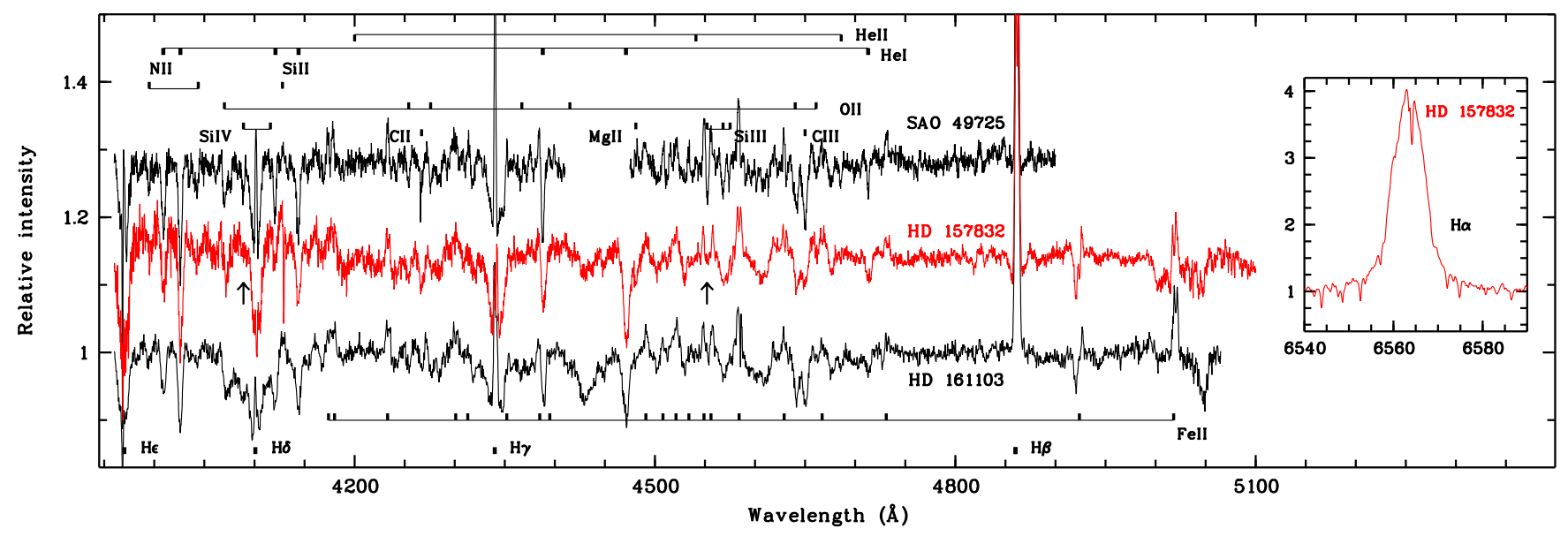

Fig. 1.- Optical spectrum of HD 157832. The spectra of the $\gamma$-Cas-like stars HD 161103 and SAO 49725 are shown for comparison (after Lopes de Oliveira et al. 2006).

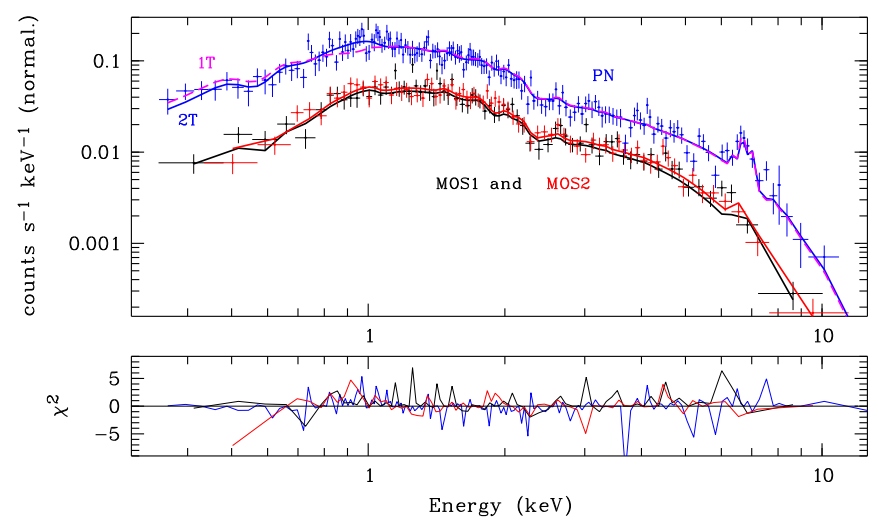

Fig. 2.- EPIC/XMM spectra of HD 157832. Solid lines represent the best 2-T model fit, while the dashed line corresponds to the 1-T model (only shown for the PN spectrum).

Table 1: X-ray spectral parameters

\begin{tabular}{lc}
\hline \hline Parameter & 2-thermal \\
\hline$N_{H}\left(10^{21} \mathrm{~cm}^{-2}\right)$ & $2.3 \pm 0.2$ \\
$k T_{\text {soft }}$ & $0.82_{-0.11}^{+0.16}$ \\
$f_{x}\left(10^{-13} \mathrm{erg} \mathrm{cm}^{-2} \mathrm{~s}^{-1}\right)$ & 1.2 \\
$\mathrm{EM}\left(10^{55} \mathrm{~cm}^{-3}\right)$ & 0.04 \\
$k T_{\text {hot }}$ & $11.25_{-1.57}^{+2.40}$ \\
$f_{x}\left(10^{-12} \mathrm{erg} \mathrm{cm}^{-2} \mathrm{~s}^{-1}\right)$ & 3.7 \\
$\mathrm{EM}\left(10^{55} \mathrm{~cm}^{-3}\right)$ & 1.1 \\
$\chi_{\nu}^{2} /$ d.o.f. & $0.94 / 319$ \\
\hline
\end{tabular}




\subsection{Timing analysis}

Timing analysis was performed on both PN eventlist and binned (10 s) light curves created by summing all EPIC cameras in the energy bands $0.3-10 \mathrm{keV}, 0.3-2 \mathrm{keV}$, and $2-10 \mathrm{keV}$. In all cases, photon arrival times were corrected to the barycenter of the solar system. The light curves were corrected for inefficiencies in the mirror-detector system and background subtracted with the EPICLCCORR/SAS task v1.4.7. We searched for short periodicities up to the Nyquist frequency of $\sim 6.81 \mathrm{~Hz}$ using the $Z_{n}^{2}$ periodogram (Buccheri et al. 1983) applied to the PN eventlist. No significant signal was found in the range of periods from $0.147 \mathrm{~s}$ to $20 \mathrm{~s}$ with a pulsed fraction larger than $14 \%(0.3-10 \mathrm{keV})$. Binned light curves were mainly used to investigate hardness ratio variations with time and intensity, and to search for "long-term" periodicities $(P>20 \mathrm{~s})$. A wealth of peaks are seen in the Scargle periodograms (Scargle 1982) with unfortunately only low statistical probabilities to be associated with a true period. Random variations are clearly seen in the X-ray light curve (see Fig. 3). The intensity can suffer an increase (decrease) of $\sim 140 \%(\sim 80 \%)$ on time scale as short as 50 s. Hardness ratios do not show correlations with the intensity of the source.

\section{Long term X-ray variations}

HD 157832 was serendipitously observed in the XMM-Newton slew survey (XMMSL1; Saxton et al. 2008) on 2004 February 26. The $0.2-12 \mathrm{keV}$ flux of $3.4 \pm 1.0 \times 10^{12} \mathrm{erg} \mathrm{s}^{-1} \mathrm{~cm}^{-2}$ detected at this occasion is consistent with that measured in 2008. Most interestingly, the star was not detected in the ROSAT all-sky survey. Scanning observations took place in August/September 1990 for a total of $\sim 400$ s (derived from the exposure maps) spread over a time interval $\gtrsim 2$ days. A faint low likelihood source, RXS J172754.1-470246, is present at only $1.2^{\prime}$ from HD 157832 and is not detected again in the XMM-Newton EPIC images. Although RXS J172754.1-470246 is located too far to be associated with the new $\gamma$-Caslike star the tail of its PSF adds some photons to the area enclosed by HD 157832. We estimated a $90 \%$ confidence upper limit of $0.02 \mathrm{cts} \mathrm{s}^{-1}$ in the $0.1-2.4 \mathrm{keV}$ ROSAT PSPC band for HD 157832 using the uplimit task (Gehrels 1986) in the XIMAGE⿴囗十⺝ package. Using WEBPimms v4.2a we find that if the star would have had the X-ray flux seen by XMMNewton at the time of the ROSAT all-sky survey, it would have been detected with a count rate of $0.055 \mathrm{cts} \mathrm{s}^{-1}$. HD 157832 was thus on average at least 2.7 times fainter in 1990 than in 2008 and in 2004 .

\footnotetext{
${ }^{2}$ http://heasarc.gsfc.nasa.gov/xanadu/ximage/ximage.html
} 


\section{Discussion}

$\gamma$-Cas-like stars display astonishingly homogeneous X-ray and optical properties (Motch et al. 2007). HD 157832 clearly exhibits all key characteristics of this class of objects, namely, an unusually hard thermal X-ray spectrum with a luminosity in the range of $\sim 10^{32-33} \mathrm{erg} \mathrm{s}^{-1}$ variable on short time scales, and on the optical side, an early B type spectrum with a dense circumstellar disc. HD 157832 is thus a new $\gamma$-Cas-like Be star.

However, ignoring the two faintest objects (USNO 0750-13549725 and SS 397; Lopes de Oliveira et al. 2006) for which only low resolution optical spectra and uncertain classification are available, all other $\gamma$-Cas analogs, HD 161103, SAO 49725, HD 110432 (Smith \& Balona 2006), HD 119682 (Safi-Harb et al. 2007), and $\gamma$-Cas itself have a B0.5e spectral type with a luminosity class III to V. HD 157832 is therefore the "coolest" $\gamma$-Cas analog identified so far.

The lack of detection in the ROSAT all-sky survey over a $\gtrsim 2$ day time interval shows that the soft (0.1-2.4 keV) X-ray luminosity of HD 157832 varied quite significantly on a time scale of several years. Variability by a factor of $\sim 3$ has been observed from $\gamma$-Cas itself in the hard (2-30 keV) band of RXTE on a time scale of 50 to 90 days (Smith et al. 2006). These modulations were accompanied by cycles in the $\mathrm{B}$ and $\mathrm{V}$ optical bands strongly suggesting that X-rays and optical variations have intimately related mechanisms. ROSAT pointed observations of $\gamma$-Cas revealed a factor 3 variability of the (0.1-2.4 keV) band flux on a time scale of a few hours. However, the average PSPC count rate changed by only a factor $\sim 1.5$ between all-sky survey and pointed observations (Haberl 1995). Therefore, our lower limit of 2.7 on the soft band long term variability of HD 157832 is in principle compatible with what is observed from $\gamma$-Cas, but could also indicate a much larger variation of the soft X-ray flux.

We did not find any information on the status of the circumstellar disc (CD) of HD 157832 close to the time of the ROSAT observations. However, both the IRAS and 2MASS mea-

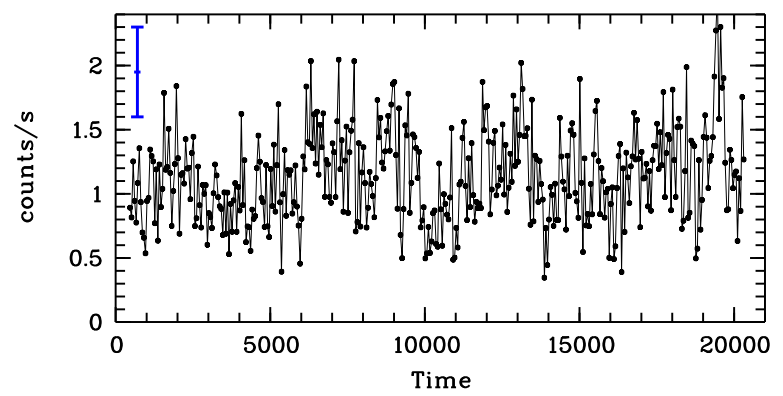

Fig. 3.- EPIC (PN+MOS1+MOS2) light curve at $0.3-10 \mathrm{keV}$. Time bin of $50 \mathrm{~s}$. A typical error bar is shown at the top-left. 
surements obtained in 1983 and 1999, respectively, show an excess of infrared emission above the stellar continuum and thus indicate the presence of a dense CD. The $16 \mathrm{yr}$ time interval between the two infrared observations is long enough to accommodate a normal B phase such as the one undergone by $\gamma$-Cas from 1942 till 1946 (Doazan et al. 1983). Alternatively, a factor 7 increase in the photo-electric absorption is enough to decrease the ROSAT PSPC count rates to $0.02 \mathrm{cts} \mathrm{s}^{-1}$ while keeping the normalization of the X-ray spectrum to its 2008 value. Lopes de Oliveira et al. (2010) report an even stronger change in the column density affecting at least $25 \%$ of the hot X-ray component of $\gamma$-Cas.

The discovery of a $\gamma$-Cas-like star displaying long term X-ray flux variations with an amplitude comparable to and maybe higher than that of $\gamma$-Cas itself opens the possibility to study in depth how X-rays correlate with emission at other wavelengths. For $\gamma$-Cas itself, X-ray, UV, and optical fluxes were correlated one with each other, but not with the orbital phase (Robinson et al. 2002). This behavior, together with the presence of migrating subfeatures in the line profiles of $\gamma$-Cas and HD 110432 (Smith \& Balona 2006) give strong support to the magnetic scenario.

It is worth noticing that in all cases where hard X-ray emission was observed from $\gamma$-Caslike stars, contemporaneous optical observations indicated the presence of a well developed circumstellar disc. Actually, the presence of the disc is a fundamental ingredient in both the "magnetic" and "binary" scenarios proposed to explain the X-ray emission of $\gamma$-Cas-like stars. While the difference in rotational velocity between the photosphere and the internal parts of the disc accounts for magnetic field winding, reconnection and particle acceleration in the magnetic model, the presence of a dense and extended CD is obviously also required in the accretion paradigm.

A multiwavelength campaign during disc dissipation, total disappearance, and disc rebuilding phases ( $\mathrm{Be} \rightarrow \mathrm{B} \rightarrow$ Be transition) will provide us with a definite answer about the role of the disc and very likely about the origin of X-ray emission in $\gamma$-Cas-like stars. For example, in the accreting scenario, the drop in X-ray luminosity associated to the disc-less episode should not be correlated with strong temperature variations of the hottest X-ray component - since this property is mainly set by the gravitational potential of the compact star. In the magnetic scenario, we expect residual X-ray emission due to active sites, tentatively similar to that observed from some magnetic O-type stars (e.g., Donati \& Landstreet 2009), and therefore hard enough to be distinguished from the soft emission usually observed in isolated massive stars.

The study of HD 157832 is relevant in at least two respects. First, the unique smaller effective temperature of HD 157832 compared to those of other $\gamma$-Cas analogs opens the possibility to study how the mass of the central star affects the X-ray emission properties 
and the evolutionary status of a putative accreting binary. Second, a monitoring of its remarkable variable and perhaps transient X-ray emission can shed light on the dependence of the X-ray emission on CD properties. Therefore, HD 157832 seems to be a promising target for unveiling the true nature of $\gamma$-Cas-like stars.

R.L.O. acknowledges financial support from the Brazilian agency FAPESP (Fundação de Amparo à Pesquisa do Estado de São Paulo) through a Young Investigator Program (numbers 2009/06295-7 and 2010/08341-3). We gratefully acknowledge the team of the Brazilian Pico dos Dias Observatory and National Astrophysics Laboratory for promptly observing our target in Director's Time and for kindly conducting the optical observations. It is our pleasure to thank Myron A. Smith for very helpful discussions and comments. We also thank the XMM-Newton User Support Group, in particular Pedro Pascual, for the help with the SASv10.

Facilities: Simbad, Aladin, Vizier.

\section{REFERENCES}

De Becker, M. et al., 2006, MNRAS, 371, 1280

Bessell, M. S., Castelli, F., \& Plez, B. 1998, A\&A, 333, 231

Buccheri, R. et al., 1983, A\&A, 128, 245

Buscombe, W. \& Foster, B. E. 1995, in MK Spectral Classifications - Twelfth General Catalog, Evanston, Illinois: Northwestern University

Cardelli, J. A., Clayton, G. C., \& Mathis, J. S. 1989, ApJ, 345, 245

Castelli, F., \& Kurucz, R. L. 2004, arXiv:astro-ph/0405087

Doazan, V., Franco, M., Sedmak, G., Stalio, R., \& Rusconi, L. 1983, A\&A, 128, 171

Donati, J.-F., \& Landstreet, J. D., 2009, A\&A Rev., 47, 333

Gehrels, N. 1986, ApJ, 303, 336

Güdel, M., \& Nazé, Y., 2009, A\&A Rev., 17, 309

Haberl, F. 1995, A\&A, 296, 685

Humphreys, R. M. \& McElroy, D. B. 1984, ApJ, 284, 565 
Jaschek, C. \& Jaschek, M. 1992, AAS, 95, 535

Kozok, J. R., 1985, A\&AS, 62, 7

Lopes de Oliveira, R., Motch, C., Haberl, F., Negueruela, I., \& Janot-Pacheco, E., 2006, A\&A, 454, 265

Lopes de Oliveira, R., Motch, C., Smith, M. A., Negueruela, I., Torrejón, J. M., 2007, A\&A, 474,983

Lopes de Oliveira, R., Smith, M. A., \& Motch, C., 2010, A\&A, 512, 22

Motch, C., Lopes de Oliveira, R., Negueruela, I., Haberl, F., \& Janot-Pacheco, E., 2007, in Active OB-Stars: Laboratories for Stellar and Circumstellar Physics, Hokkai-Gakuen University, Sapporo, Japan, ed. S. Stefl, S. P. Owocki, \& A. T. Okazaki, San Francisco: ASP Conf. Ser., 361, 117

Rakowski, C. E., Schulz, N. S., Wolk, S. J., \& Testa, P., ApJ, 649, 111

Robinson, R. D., Smith, M. A., \& Henry, G. W., 2002, ApJ, 575, 435

Safi-Harb, S. et al., 2007, ApJ, 659, 407

Saxton, R. D., et al. 2008, A\&A, 480, 611

Scargle, J. D. 1982, ApJ, 263, 835

Smith, M. A., \& Robinson, R. D., 2003, in ASP Conf. Ser. 292, Interplay between Periodic, Cyclic, and Stochastic Variability, ed. C. Sterken (San Francisco: ASP), 263

Smith, M. A. et al., 2004, ApJ, 600, 972

Smith, M. A., Henry, G. W., \& Vishniac, E., 2006, ApJ, 647, 1375

Smith, M. A., \& Balona, L. 2006, ApJ, 640, 491

Steele, I. A., Negueruela, I., \& Clark, J. S., 1999, A\&AS, 137, 147

Thackeray, A. D., Tritton, S. B., Walker, E. N., 1973, MmRAS, 77, 199 